\title{
COMPLETE SYNCHRONIZATION OF KURAMOTO OSCILLATORS WITH HIERARCHICAL LEADERSHIP*
}

\author{
SEUNG-YEAL HA ${ }^{\dagger}$ AND ZHUCHUN LI ${ }^{\ddagger}$
}

\begin{abstract}
We study the asymptotic emergence of the complete synchronization of Kuramoto oscillators with hierarchical leadership. We show that asymptotic complete frequency synchronization occurs for some class of initial phase configurations, when Kuramoto oscillators are scattered on the nodes of a directed graph with a hierarchical leadership topology and the coupling strength is sufficiently large. This generalizes previous studies on the complete synchronization of Kuramoto oscillators on connected and symmetric graphs. We also provide several numerical results to confirm our analytical results.
\end{abstract}

Key words. Kuramoto oscillators, hierarchical leadership, complete synchronization.

AMS subject classifications. 34C15, 92D25.

\section{Introduction}

The synchronization of many weakly coupled oscillators often appears in natural systems, e.g., two pendulum clocks suspended from the same bar, the flashing of fireflies, the singing of crickets and hand clapping by audiences in a concert hall, etc. (see $[1,5]$ ), which were first reported by C. Huygens in the scientific literature. The rigorous mathematical study of these systems was started forty years ago by the two pioneers Winfree [39] and Kuramoto [24]. They introduced simple phase models for weakly coupled oscillators and showed how the synchronized behavior of their phase models can emerge from the competing mechanisms of intrinsic randomness and nonlinear coupling. Recently, the study of the synchronization process of weakly coupled oscillators has emerged as an active research area because of its diverse applications in areas such as social opinion formation, formation control of unmanned aerial vehicles (UAV), and the control of automatic robot systems etc. ([31, 38]). These applications can be reformulated as a synchronization process of weakly coupled oscillators in complex networks, and the crucial component when understanding such a synchronization process is elucidating the interplay between the network structure (topology) and dynamics of oscillators. For a detailed survey of this issue, please refer to [4].

In this study, we investigate the emergence of the asymptotic complete synchronization (ACS) of Kuramoto oscillators, where the underlying network has a hierarchical leadership (HL) structure. The complete synchronization problem is to look for sufficient conditions for the initial phase configurations and parameters (e.g. the natural frequency distribution and coupling strength) leading to ACS. For a detailed discussion, we refer to survey papers and books $[1,7,23,36]$.

We next introduce the Kuramoto model with an HL structure. Consider a digraph $\mathcal{D}$ consisting of a vertex-set $\mathcal{V}:=\{1, \ldots, N\}$ and an edge set $\mathcal{A}$. Let $\psi_{i j} \geq 0$ be the capacity of the edge connecting from the $i$-th vertex to the $j$-th vertex. Suppose that Kuramoto oscillators are located at the vertices and the interactions between them are

*Received: October 6, 2012; accepted (in revised form): March 25, 2013. Communicated by Paul Milewski.

${ }^{\dagger}$ Department of Mathematical Sciences, Seoul National University, Seoul 151-747, South Korea (syha@snu.ac.kr).

${ }_{\ddagger}^{\ddagger}$ Department of Mathematics, Harbin Institute of Technology, Harbin 150001, P.R. China (lizhuchun@gmail.com). Current address: Department of Mathematical Sciences, Seoul National University, Seoul 151-747, South Korea. 
registered in the capacity (or adjacent) matrix $\psi=\left(\psi_{i j}\right)$. Kuramoto oscillators can be visualized as active point rotors moving on the unit circle $\mathbb{S}^{1} \subset \mathbb{C}$. More specifically, let $x_{i}$ be the position of the $i$-th rotor and we rewrite $x_{i}=e^{\sqrt{-1} \theta_{i}}, \theta_{i} \in \mathbb{R}$ via the polar coordinate. Then the dynamics of Kuramoto oscillators in a network with HL structure is governed by the following ODE system in $\mathbb{R}^{N}$ :

$$
\begin{aligned}
\dot{\theta}_{i} & =\Omega_{i}+K \sum_{j=1}^{N} \psi_{j i} \sin \left(\theta_{j}-\theta_{i}\right), \quad t>0, \\
\theta_{i}(0) & =\theta_{i 0}, \quad i=1, \ldots, N,
\end{aligned}
$$

where $K>0$ is the uniform coupling strength, and $\Omega_{i}$ represents the intrinsic natural frequency of the $i$-th oscillator drawn from some distribution function $g=g(\Omega)$.

For the complete graph $\psi_{i j}=\frac{1}{N}$, Ermentrout [16] found a critical coupling strength, when all oscillators became phase-locked, independent of the number of oscillators. The linear stability of the phase-locked state has been studied in several papers, e.g. van Hemmen and Wreszinski [37], Mirollo and Strogatz [27, 28, 29], Aeyels and Rogge [2], Bonilla, Neu, and Spigler [6], and De Smet and Aeyels [13] using tools such as Lyapunov functions and spectral graph theory. Recently the ACS problem on the complete graph has been systematically studied in $[8,9,10,14,17,19,21,32]$. On the other hand, Jadbabaie et al [22] addressed ACS of Kuramoto oscillators on undirected connected graphs by combining the techniques from control theory and graph theory.

The main result of this paper is to establish ACS for asymmetric and directed graphs with HL structure (see Definition 2.2 in Section 2). In general, when the capacity matrix $\psi=\left(\psi_{i j}\right)$ is not symmetric, the standard energy estimate does not work due to the nonconservation of overall phase, so the previously mentioned methods in $[9,14]$ cannot be applied to the current situation. Nevertheless even if the capacity matrix $\psi$ is asymmetric, it is triangular, and this enables us to use the induction method to derive the exponential decay of the deviations from the phase of the global leader.

The rest of the paper consists of five sections. In Section 2, we present definitions of the ACS and HL, and we provide several estimates on Adler type equations [3, 8] as well as discussing Lyapunov functions to be used in later sections. In sections 3 and 4, we provide rigorous ACS estimates for identical and nonidentical Kuramoto oscillator groups with HL. In Section 5, we provide several numerical results consistent with the analytical results in previous sections. Finally Section 6 is devoted to the summary of the main results.

\section{Preliminaries}

In this section, we first provide the definitions of the ACS and HL topology. Then we briefly present several key estimates on Adler type equations and discuss Lyapunov functions which will be crucially used in later sections. First we provide a definition of ACS for the system (1.1).

Definition 2.1. Let $\mathcal{G}:=\left\{\theta_{i}\right\}$ be the group of Kuramoto oscillators whose dynamics is governed by the system (1.1). The system $\mathcal{G}$ has ACS if and only if all transversal frequency differences converge to zero asymptotically:

$$
\lim _{t \rightarrow \infty} \max _{1 \leq i, j \leq N}\left|\dot{\theta}_{i}(t)-\dot{\theta}_{j}(t)\right|=0 .
$$


For a given coupling strength, oscillators with small natural frequencies relative to the coupling strength will be locked, whereas oscillators with large natural frequencies will move around the circle without locking. As the coupling strength $K$ is increased from zero to some critical value, the number of phase-locked oscillators will increase accordingly, whereas when it reaches to some critical coupling strength, all the oscillators will be phase-locked, i.e., their relative phases will become constant. We refer to this state as the "complete synchronization". It is well known [17] that the complete synchronization cannot occur within finite time, so the complete synchronization should be understood in a time-asymptotic sense.

Definition 2.2. The adjacent capacity matrix $\psi=\left[\psi_{i j}\right]$ has an "HL" structure if and only if the matrix $\psi$ satisfies the following two conditions:

1. Followers are influenced only by leaders, i.e.,

$$
\psi_{j i}>0 \Longrightarrow j<i
$$

2. The leader set of the $i$-th oscillator $\mathcal{L}(i):=\left\{j: 1 \leq j \leq i, \psi_{j i}>0\right\}$ is not empty for all $i>1$.

REMARK 2.3.

1. The concept of HL was first introduced by J. Shen [34] for both the discrete and continuous-time Cucker-Smale flocking models [12], while Cucker and Dong [11] later improved the flocking result for a discrete-time flocking model [34].

2. The adjacent matrix $\psi$ with an HL structure is a triangular matrix, and hence $\psi$ is asymmetric.

3. Note that the first oscillator with phase $\theta_{1}$ is not influenced by other oscillators, so it is invariant under the flow (1.1):

$$
\dot{\theta}_{1}=\Omega_{1}, \quad \text { i.e., } \quad \theta_{1}(t)=\theta_{10}+\Omega t, \quad t \geq 0 .
$$

However the phase state of the first oscillator affects the rest of oscillators. Therefore, we call it the global leader.

For the system (1.1) with a symmetric and connected topology, the standard energy method based on the $\ell_{2}$-norm of the fluctuations does work for the complete synchronization problem as in $[18,20]$. In contrast, for the system (1.1) with a general asymmetric network structure, there is no general systematic method for ACS. In the following analysis, the hierarchical structure plays a key role in the ACS problem. In [25], Li and Xue introduced another interesting interaction topology called a rooted leadership (in short RL), which is more general than HL in the sense that the hierarchy is dropped in RL.

2.1. A generalized Adler equation. In this part, we present several estimates for a generalized Adler equation. These estimates will be used in the ACS estimates in sections 3 and 4 .

Consider a generalized Adler equation:

$$
\begin{aligned}
\dot{y} & =\varepsilon(t)-\eta(t) \sin y, \quad t>0, \quad y(0)=y_{0}, \\
|\varepsilon(t)| & \leq A_{*} e^{-B_{*} t}, \quad \inf _{t \geq 0} \eta(t) \geq \eta_{*}>0,
\end{aligned}
$$

where $A_{*}, B_{*}$, and $\eta_{*}$ are positive constants, while $\varepsilon(t)$ and $\eta(t)$ are continuous functions of $t$. 
LEMMA 2.4. Let $y=y(t)$ be a solution to (2.1) satisfying the a priori condition

$$
\sup _{t \geq 0}|y(t)| \leq \alpha, \quad \alpha \in(0, \pi)
$$

Then $y$ decays to zero exponentially fast:

$$
|y(t)| \leq A e^{-B t}, \quad \forall t \geq 0
$$

where $A$ and $B$ are given by the following relations:

$$
A:=2 \max \left\{|| y_{0}\left|-\frac{A_{*}}{\frac{\eta_{*} \sin \alpha}{\alpha}}\right|,\left|\frac{A_{*}}{\frac{\eta_{*} \sin \alpha}{\alpha}-B_{*}}\right|\right\}, \quad B:=\min \left\{\frac{\eta_{*} \sin \alpha}{\alpha}, B_{*}\right\} .
$$

Proof. We multiply $(2.1)$ by $\operatorname{sgn}(y(t))$ and use the fact that

$$
\operatorname{sgn}(y) \sin y=\sin |y| \geq \frac{\sin \alpha}{\alpha}|y|, \quad \text { for }|y|<\alpha
$$

to derive the Gronwall's inequality for $|y|$ :

$$
\frac{d|y|}{d t} \leq-\eta \frac{\sin \alpha}{\alpha}|y|+|\varepsilon| \leq-\eta_{*} \frac{\sin \alpha}{\alpha}|y|+A_{*} e^{-B_{*} t}
$$

where we used the relations

$$
|\varepsilon(t)| \leq A_{*} e^{-B_{*} t}, \quad \inf _{t \geq 0} \eta(t) \geq \eta_{*}>0 .
$$

Then we apply the standard Gronwall's lemma for (2.2) to obtain

$$
|y(t)| \leq\left|y_{0}\right| e^{-\frac{\eta_{*} \sin \alpha}{\alpha} t}+\frac{A_{*}}{\frac{\eta_{*} \sin \alpha}{\alpha}-B_{*}}\left(e^{-B_{*} t}-e^{-\frac{\eta_{*} \sin \alpha}{\alpha} t}\right) .
$$

This implies the desired result.

In the following lemma, we consider a simple situation where the coefficients functions $\varepsilon(t)$ and $\eta(t)$ in (2.1) are simply positive constants. In this case, we can find an implicit representation of the solutions by integrating the Adler equation:

$$
\dot{y}=\varepsilon-\eta \sin y, \quad t>0, \quad y(0)=y_{0}
$$

LEMMA 2.5. The solution $y=y(t)$ to (2.3) satisfies the following representations:

1. Case $1(0 \leq \varepsilon<\eta)$ :

$$
t \sqrt{\eta^{2}-\varepsilon^{2}}=\log \left|\frac{\eta \tan \frac{y(t)}{2}-\eta-\sqrt{\eta^{2}-\varepsilon^{2}}}{\varepsilon \tan \frac{y(t)}{2}-\eta+\sqrt{\eta^{2}-\varepsilon^{2}}}\right|-\log \left|\frac{\eta \tan \frac{y_{0}}{2}-\eta-\sqrt{\eta^{2}-\varepsilon^{2}}}{\varepsilon \tan \frac{y_{0}}{2}-\eta+\sqrt{\eta^{2}-\varepsilon^{2}}}\right| .
$$

2. Case $2(\varepsilon=\eta)$ :

$$
y(t)=2 \tan ^{-1}\left[\frac{2 \tan \frac{y_{0}}{2}+\varepsilon\left(1-\tan \frac{y_{0}}{2}\right) t}{2+\varepsilon\left(1-\tan \frac{y_{0}}{2}\right) t}\right] .
$$


3. Case $3(\varepsilon>\eta)$ :

$$
y(t)=2 \tan ^{-1}\left[\left\{\frac{\sqrt{\varepsilon^{2}-\eta^{2}}}{\varepsilon} \tan \left\{\frac{t}{2} \sqrt{\varepsilon^{2}-\eta^{2}}+\tan ^{-1}\left(\frac{\varepsilon \tan \frac{y_{0}}{2}-\eta}{\sqrt{\varepsilon^{2}-\eta^{2}}}\right)\right\}+1\right\} .\right.
$$

Proof. The proof can be found in Appendix D of [8].

REMARK 2.6.

1. Note that in Case 1, by the simple phase analysis of Adler's equation (2.3), it is easy to see that if the initial datum $y_{0} \in\left(-\frac{\pi}{2}, \frac{\pi}{2}\right)$, then the solution $y=y(t)$ to $(2.3)$ approaches the equilibrium solution $y_{e}:=\sin ^{-1} \frac{\varepsilon}{\eta} \in\left[0, \frac{\pi}{2}\right)$ monotonically. Moreover, it follows from the implicit representation formula that if the initial data $y_{0}$ is sufficiently close to the stable equilibrium $y_{e}$, then the solution $y(t)$ converges to the equilibrium $y_{e}$ exponentially fast, more precisely,

$$
\left|y(t)-y_{e}\right| \leq C e^{-\sqrt{\eta^{2}-\varepsilon^{2}} t}, \quad \text { whenever }\left|y_{0}-y_{e}\right| \ll 1 .
$$

2. For the case where $\varepsilon<0$ and $|\varepsilon|<\eta$, we can obtain analogous results except that the stable equilibrium $y_{e}:=\sin ^{-1} \frac{\varepsilon}{\eta}$ lies in the interval $\left(-\frac{\pi}{2}, 0\right)$ rather than $\left(0, \frac{\pi}{2}\right)$.

2.2. Lyapunov functions. In this part, we discuss the Lyapunov functions measuring the complete synchronization in Definition 2.1.

The diameters of the frequency sets $\left\{\omega_{i}:=\dot{\theta}_{i}\right\}$ and $\left\{\Omega_{i}\right\}$ are defined as

$$
D(\omega):=\max _{i, j}\left|\omega_{i}-\omega_{j}\right|, \quad D(\Omega):=\max _{i, j}\left|\Omega_{i}-\Omega_{j}\right| .
$$

For the ACS analysis, we will study the time evolution for the function $D(\omega(\cdot))$, and show that $D(\omega(\cdot))$ approaches to zero asymptotically. Thus the function $D(\omega)$ serves as a Lyapunov function for the ACS problem.

On the other hand, van Hemmen et al [37] proposed a Lyapunov function for the original Kuramoto model with identical oscillators and an all-to-all coupling:

$$
H=-\frac{K}{2 N} \sum_{i, j=1}^{N} \cos \left(\theta_{i}-\theta_{j}\right)
$$

Then the original Kuramoto model can be written as a gradient system with analytic potential $H$ :

$$
\frac{\partial H}{\partial \theta_{i}}=-\frac{K}{N} \sum_{j=1}^{N} \sin \left(\theta_{j}-\theta_{i}\right)
$$

In [22], Jadbabaie et al studied the Kuramoto model on undirected graphs by considering a Lyapunov function based on the square of the order parameter given by

$$
r^{2}=\frac{N^{2}-2 e+2 \mathbf{1}_{e}^{\top} \cos \left(B^{\top} \theta\right)}{N^{2}},
$$

where $e$ is the number of edges and $B$ is the incidence matrix of the graph. Of course, the key part, $\mathbf{1}_{e}^{\top} \cos \left(B^{\top} \theta\right)$, possesses the advantage of the potential function $H$. However, it is impossible to construct such a potential function for the Kuramoto model with an asymmetric network topology, such as an HL interaction. To show the 
necessity, let us suppose that the Kuramoto oscillators system can be reformulated as a gradient system with an analytic potential $f$, i.e.,

$$
\partial_{t} \theta=-\nabla_{\theta} f(\theta) .
$$

Then $f$ must satisfy

$$
\partial_{\theta_{k}} \partial_{\theta_{l}} f=\partial_{\theta_{l}} \partial_{\theta_{k}} f, \quad l \neq k,
$$

i.e.,

$$
-K \psi_{k l} \cos \left(\theta_{k}-\theta_{l}\right)=-K \psi_{l k} \cos \left(\theta_{l}-\theta_{k}\right), \quad l \neq k .
$$

This yields $\psi_{l k}=\psi_{k l}, l \neq k$, i.e., $\psi$ is symmetric.

\section{ACS for identical oscillators}

In this section, we present ACS for identical Kuramoto oscillators with an HL structure. First, we show that the phase fluctuations around $\theta_{1}$ are uniformly bounded by $\pi$, once the diameter of the set of initial phases is strictly less than $\pi$. Then, from this rough a priori information, we use the method of induction to show that the fluctuations in fact decay to zero exponentially fast. This induction argument crucially depends on the triangular structure of the capacity matrix $\psi=\left[\psi_{i j}\right]$.

We would like to remark that the synchronization result for identical oscillators appears in other literatures $[15,26,30,33]$. However, we prefer to present a simple proof by using the method of induction, which provides a motivation for the analysis for non-identical oscillators in the next section.

3.1. Existence of a positively invariant set. In this part, we consider the complete phase synchronization of Kuramoto oscillators with the same natural frequency $\Omega$ :

$$
\dot{\theta}_{i}=\Omega+K \sum_{j=1}^{N} \psi_{j i} \sin \left(\theta_{j}-\theta_{i}\right) .
$$

Note that the first oscillator $\theta_{1}$ is not influenced by other oscillators, so that it rotates on the circle with a constant speed $\Omega$ :

$$
\dot{\theta}_{1}=\Omega, \quad \theta_{1}(t)=\theta_{10}+\Omega t .
$$

When the asymptotic complete phase synchronization occurs, i.e.,

$$
\lim _{t \rightarrow \infty}\left|\theta_{i}(t)-\theta_{j}(t)\right|=0,
$$

the phases of all the oscillators should approach to $\theta_{1}$ asymptotically, so it would be reasonable to consider the fluctuations around $\theta_{1}$ :

$$
\hat{\theta}_{i}:=\theta_{i}-\theta_{1}, \quad i=1, \ldots, N .
$$

Obviously the fluctuation $\hat{\theta}_{i}$ satisfies

$$
\dot{\hat{\theta}}_{i}=K \sum_{j=1}^{N} \psi_{j i} \sin \left(\hat{\theta}_{j}-\hat{\theta}_{i}\right), \quad i=2, \ldots, N
$$


We next define the extremal phase fluctuations for a set of phases $\left\{\theta_{i} \in \mathbb{R}\right\}$ :

$$
\hat{\theta}_{m}:=\min _{1 \leq i \leq N} \hat{\theta}_{i}, \quad \hat{\theta}_{M}:=\max _{1 \leq i \leq N} \hat{\theta}_{i} .
$$

Then the phase diameter is the difference of the extremal phase fluctuations:

$$
D(\theta):=\hat{\theta}_{M}-\hat{\theta}_{m} .
$$

Note that the solution to system (3.2) is real-analytic and the extremal phase fluctuations $\hat{\theta}_{M}(t)$ and $\hat{\theta}_{m}(t)$ are Lipschitz continuous in time, and therefore $D(\theta(t))$ is Lipschitz continuous and non-differentiable points are isolated and finite in any finitetime interval by using the same argument as in [17, Section 2.2]. Therefore, there exist at most countably many collision times $0 \leq t_{0}<t_{1}<\cdots$ and hence $D(\theta(t))$ is differentiable in each time interval $\left(t_{k-1}, t_{k}\right)$.

For any $t \geq 0$, we set

$$
I_{\min }(t):=\underset{1 \leq i \leq N}{\arg \min } \hat{\theta}_{i}(t) \quad \text { and } \quad I_{\max }(t):=\underset{1 \leq i \leq N}{\arg \max } \hat{\theta}_{i}(t),
$$

then the upper Dini derivative of $D(\theta(t))$ at time $t$ along the system (3.1) or (3.2) is given by [26, Lemma 2.2]

$$
D^{+} D(\theta(t))=\limsup _{h \downarrow 0} \frac{D(\theta(t+h))-D(\theta(t))}{h}=\dot{\hat{\theta}}_{\bar{M}}(t)-\dot{\hat{\theta}}_{\bar{m}}(t)
$$

where $\dot{\hat{\theta}}_{\bar{M}}(t)=\max \left\{\dot{\hat{\theta}}_{i}(t): i \in I_{\max }(t)\right\}$ and $\dot{\hat{\theta}}_{\bar{m}}(t)=\min \left\{\dot{\hat{\theta}}_{i}(t): i \in I_{\min }(t)\right\}$. Since the function $D(\theta(t))$ is differentiable in time interval $\left(t_{k-1}, t_{k}\right)$, the upper Dini derivative coincides with the classic derivative, i.e., $\dot{D}(\theta(t))=\dot{\hat{\theta}}_{\bar{M}}(t)-\dot{\hat{\theta}}_{\bar{m}}(t), t \in\left(t_{k-1}, t_{k}\right), k=$ $1,2, \ldots$ Throughout this paper, we use the usual notations $\left(\dot{D}(\theta(t))\right.$ and $\left.\frac{d D(\theta(t))}{d t}\right)$ to denote the classic derivative for almost all time $t$ and the upper Dini derivative at collision time $t_{k}$.

We next define an admissible class of initial configurations and extremal phase fluctuations:

$$
\mathcal{S}_{1}:=\left\{\theta=\left(\theta_{1}, \ldots, \theta_{N}\right) \in \mathbb{R}^{N}: D(\theta)<\pi\right\} .
$$

We have the following lemma.

Lemma 3.1. Let $\left\{\theta_{i}\right\}_{i=1}^{N}$ be the global smooth solution to (3.1) with the initial phase $\theta_{0}$ :

$$
\theta_{0}=\left(\theta_{10}, \ldots, \theta_{N 0}\right) \in \mathcal{S}_{1}
$$

Then we have

$$
\theta(t) \in \mathcal{S}_{1}, \quad \forall t \geq 0
$$

Proof. - Case $1\left(D\left(\theta_{0}\right)=0\right)$ : In this case, we have

$$
\hat{\theta}_{10}=\cdots=\hat{\theta}_{N 0},
$$

i.e., $\hat{\theta}_{0}$ is in the equilibrium state of the system (3.2). Hence we have

$$
\hat{\theta}_{1}(t)=\cdots=\hat{\theta}_{N}(t), \quad \text { i.e., } \quad D(\theta(t))=0 .
$$


- Case $2\left(0<D\left(\theta_{0}\right)<\pi\right)$ : By the continuity of $D(\theta(t))$, because $D\left(\theta_{0}\right)<\pi$, there exists $\delta>0$ such that

$$
D(\theta(t))<\pi, \quad t \in[0, \delta) .
$$

We now define a set

$$
\mathcal{T}_{1}:=\left\{T>0: \theta(t) \in \mathcal{S}_{1}, \quad \forall t \in[0, T)\right\} .
$$

Clearly $\mathcal{T}_{1}$ is not empty, because $\delta \in \mathcal{T}_{1}$. We now claim that

$$
T_{1}^{*}:=\sup \mathcal{T}_{1}=\infty \text {. }
$$

The proof of the claim: Suppose this is not the case, i.e., $T_{1}^{*}<\infty$. Then there exists a sequence of finite numbers of collision times $\left\{t_{i}\right\}_{i=0}^{n}$ such that

$$
\begin{aligned}
& 0=t_{0}<t_{1}<\cdots<t_{n}=T_{1}^{*} \text { and } \\
& D(\theta(\cdot)) \text { is differentiable on each time interval }\left(t_{i-1}, t_{i}\right), i=1, \ldots, n .
\end{aligned}
$$

For each interval $\left(t_{i-1}, t_{i}\right)$, we have

$$
\frac{d D(\theta(t))}{d t}=K \sum_{j=1}^{N} \psi_{\bar{M} j} \sin \left(\hat{\theta}_{j}-\hat{\theta}_{\bar{M}}\right)-K \sum_{j=1}^{N} \psi_{\bar{m} j} \sin \left(\hat{\theta}_{j}-\hat{\theta}_{\bar{m}}\right) \leq 0,
$$

where we used the fact that

$$
-\pi \leq \hat{\theta}_{j}-\hat{\theta}_{\bar{M}} \leq 0 \quad \text { and } \quad 0 \leq \hat{\theta}_{j}-\hat{\theta}_{\bar{m}} \leq \pi, \quad \forall t \in[0, T) .
$$

Therefore $D(\theta(t))$ is nonincreasing in $\left[0, T_{1}^{*}\right)$, in particular

$$
D\left(\theta\left(T_{1}^{*}\right)\right)=\lim _{t \rightarrow T_{1}^{*}-} D(\theta(t)) \leq D\left(\theta_{0}\right)<\pi .
$$

Then again, based on the continuity of $D(\theta(t))$, there exists $\delta^{\prime}>0$ such that

$$
D(\theta(t))<\pi, \quad t \in\left[0, T_{1}^{*}+\delta^{\prime}\right), \quad \text { i.e., } \quad T_{1}^{*}+\delta^{\prime} \in \mathcal{T}_{1} .
$$

This contradicts to the statement that $T_{1}^{*}=\sup \mathcal{T}_{1}$. Therefore $T_{1}^{*}=\infty$ and we have

$$
D(\theta(t))<\pi, \quad t>0 .
$$

3.2. Exponential complete synchronization. In this part, we reinforce the rough estimate $D(\theta)<\pi$ to get the refined exponential decay estimate of $D(\theta(t))$ using the induction method.

THEOREM 3.2. Let $\left\{\theta_{i}\right\}_{i=1}^{N}$ be the global solution to (3.1) with the initial configuration $\theta_{0} \in \mathcal{S}_{1}$. Then for any $0<\delta \ll 1$, there exist positive constants $A_{N}=$ $A_{N}\left(\psi, K, \delta, \theta_{0}\right), B_{N}=B_{N}\left(\psi, K, \delta, \theta_{0}\right)$, and $T_{N}=T_{N}\left(\psi, K, \delta, \theta_{0}\right) \geq 0$ such that

$$
D(\theta(t)) \leq A_{N} e^{-B_{N} t}, \quad t \geq T_{N}
$$

Proof. Note that

$$
D(\theta(t)) \leq 2 \max _{1 \leq i \leq N}\left|\hat{\theta}_{i}(t)\right| \quad \text { and } \quad \hat{\theta}_{1}(t)=0, \quad t \geq 0
$$


Thus, it suffices to show that

$$
\max _{2 \leq i \leq N}\left|\hat{\theta}_{i}(t)\right| \leq \frac{A_{N}}{2} e^{-B_{N} t}, \quad t \geq T_{N} .
$$

We prove the estimate (3.3) by the induction method.

- (Initial step): It follows from (3.2) that

$$
\dot{\hat{\theta}}_{2}=-K \psi_{12} \sin \hat{\theta}_{2}, \quad t>0, \quad \hat{\theta}_{2}(0)=\hat{\theta}_{20} .
$$

We now use the fact

$$
\left|\hat{\theta}_{2}(t)\right| \leq D\left(\theta_{0}\right)<\pi, t \geq 0
$$

to derive

$$
\frac{d\left|\hat{\theta}_{2}\right|}{d t} \leq-K \psi_{12} \frac{\sin D\left(\theta_{0}\right)}{D\left(\theta_{0}\right)}\left|\hat{\theta}_{2}\right| .
$$

Thus we have an exponential decay of $\hat{\theta}_{2}$ :

$$
\left|\hat{\theta}_{2}(t)\right| \leq\left|\hat{\theta}_{20}\right| e^{-\frac{K \psi_{12} \sin D\left(\theta_{0}\right)}{D\left(\theta_{0}\right)} t}, \quad t \geq 0
$$

We set

$$
A_{2}:=2\left|\hat{\theta}_{20}\right|, \quad B_{2}:=\frac{K \psi_{12} \sin D\left(\theta_{0}\right)}{D\left(\theta_{0}\right)}, \quad T_{2}:=0
$$

- (Inductive step): Suppose that the result holds for the sub-group $\{1,2, \ldots, k-1\}$ with $k \geq 2$, i.e., there exist $A_{k-1}$ and $B_{k-1}$ such that

$$
\left|\hat{\theta}_{i}(t)\right| \leq \frac{A_{k-1}}{2} e^{-B_{k-1} t}, \quad t \geq T_{k-1}, \quad i=1, \ldots, k-1 .
$$

Then it follows from (3.2) that

$$
\begin{aligned}
\dot{\hat{\theta}}_{k} & =K \sum_{j \in \mathcal{L}(k)} \psi_{j k} \sin \left(\hat{\theta}_{j}-\hat{\theta}_{k}\right) \\
& =K\left(\sum_{j \in \mathcal{L}(k)} \psi_{j k} \sin \hat{\theta}_{j}\right) \cos \hat{\theta}_{k}-K\left(\sum_{j \in \mathcal{L}(k)} \psi_{j k} \cos \hat{\theta}_{j}\right) \sin \hat{\theta}_{k},
\end{aligned}
$$

where $\mathcal{L}(k)$ is the leader set of the $k$-th oscillator as defined in Definition 2.2. Note that the leader set $\mathcal{L}(k)$ satisfies

$$
\mathcal{L}(k) \subset\{1, \cdots, k-1\} .
$$

To apply Lemma 2.4, we set

$$
\varepsilon(t):=K\left(\sum_{j \in \mathcal{L}(k)} \psi_{j k} \sin \hat{\theta}_{j}(t)\right) \cos \hat{\theta}_{k}(t), \quad \eta(t):=K\left(\sum_{j \in \mathcal{L}(k)} \psi_{j k} \cos \hat{\theta}_{j}(t)\right) .
$$


Then we use (3.5) to find

$$
|\varepsilon(t)| \leq K\left(\sum_{j \in \mathcal{L}(k)} \psi_{j k}\left|\hat{\theta}_{j}\right|\right) \leq \frac{K A_{k-1}}{2}\left(\sum_{j \in \mathcal{L}(k)} \psi_{j k}\right) e^{-B_{k-1} t}, \quad t \geq T_{k-1} .
$$

On the other hand, due to (3.5), for any $\delta>0$, there exists

$$
T_{k}:=\max \left\{\frac{1}{B_{k-1}} \ln \left(\frac{A_{k-1}}{2 \delta}\right), T_{k-1}\right\}>0
$$

such that

$$
\left|\hat{\theta}_{j}(t)\right| \leq \delta, \quad t \geq T_{k}
$$

This yields

$$
\eta(t) \geq K \cos \delta\left(\sum_{j \in \mathcal{L}(k)} \psi_{j k}\right), \quad t \geq T_{k}
$$

We also note from Lemma 3.1 that

$$
\left|\hat{\theta}_{k}(t)\right| \leq D(\theta(t)) \leq D\left(\theta_{0}\right)<\pi, \quad t \geq T_{k} .
$$

Note that (3.6)-(3.10) satisfy the framework of Lemma 2.4. Hence, it follows from Lemma 2.4 that we have

$$
\left|\hat{\theta}_{k}(t)\right| \leq \frac{A_{k}}{2} e^{-B_{k} t}, \quad t \geq T_{k}
$$

where $A_{k}$ and $B_{k}$ are given by the following iterative relations:

$$
\begin{aligned}
& A_{k}:=4 \max \left\{\left|\hat{\theta}_{k}\left(T_{k}\right)\right|-\frac{\frac{K A_{k-1}}{2} \sum_{j \in \mathcal{L}(k)} \psi_{j k}}{K \cos \delta \sum_{j \in \mathcal{L}(k)} \psi_{j k} \frac{\sin D\left(\theta_{0}\right)}{D\left(\theta_{0}\right)}}|,| \frac{\frac{K A_{k-1}}{2} \sum_{j \in \mathcal{L}(k)} \psi_{j k}}{K \cos \delta \sum_{j \in \mathcal{L}(k)} \psi_{j k} \frac{\sin D\left(\theta_{0}\right)}{D\left(\theta_{0}\right)}-B_{k-1}} \mid\right\}, \\
& B_{k}:=\min \left\{K \cos \delta \sum_{j \in \mathcal{L}(k)} \psi_{j k} \frac{\sin D\left(\theta_{0}\right)}{D\left(\theta_{0}\right)}, B_{k-1}\right\} .
\end{aligned}
$$

Note that the decay rate $B_{k}$ is non-increasing, i.e., $B_{k} \leq B_{k-1}$. Therefore (3.11) holds for any $k \geq 2$.

REMARK 3.3. Note that as $\delta \rightarrow 0$, the decay rate $B_{k}$ in (3.3) satisfies the relation

$$
\begin{aligned}
B_{k}:=\min \{ & \left.K \cos \delta \sum_{j \in \mathcal{L}(k)} \psi_{j k} \frac{\sin D\left(\theta_{0}\right)}{D\left(\theta_{0}\right)}, B_{k-1}\right\} \\
& \longrightarrow \quad B_{k}:=\min \left\{K \sum_{j \in \mathcal{L}(k)} \psi_{j k} \frac{\sin D\left(\theta_{0}\right)}{D\left(\theta_{0}\right)}, B_{k-1}\right\},
\end{aligned}
$$

i.e., asymptotically as $\delta \rightarrow 0$, the decay rate $B_{k}$ should only depend on $K,\left[\psi_{i j}\right]$, and $\theta_{0}$.

\section{ACS for nonidentical oscillators}

In this section, we present a complete synchronization estimate for nonidentical Kuramoto oscillators. Basically, we will follow the same method as in previous section, i.e., we first show that the existence of a positively invariant set, and then we derive a decay estimate for the frequency by differentiating the Kuramoto model. 
4.1. A three-oscillator system. As a motivation, we first consider a threeoscillator system under the HL topology:

$$
\begin{aligned}
& \dot{\theta}_{1}=\Omega_{1}, \quad t>0, \\
& \dot{\theta}_{2}=\Omega_{2}+K \psi_{12} \sin \left(\theta_{1}-\theta_{2}\right), \\
& \dot{\theta}_{3}=\Omega_{3}+K \psi_{13} \sin \left(\theta_{1}-\theta_{3}\right)+K \psi_{23} \sin \left(\theta_{2}-\theta_{3}\right),
\end{aligned}
$$

subject to initial data

$$
\left(\theta_{1}, \theta_{2}, \theta_{3}\right)(0)=\left(\theta_{10}, \theta_{20}, \theta_{30}\right)
$$

We set

$$
\varphi_{1}:=\theta_{1}-\theta_{2}, \quad \varphi_{2}:=\theta_{2}-\theta_{3}, \quad \Delta_{1}:=\Omega_{1}-\Omega_{2}, \quad \Delta_{2}:=\Omega_{2}-\Omega_{3} .
$$

Then we have

$$
\theta_{1}-\theta_{3}=\varphi_{1}+\varphi_{2}
$$

It is easy to see that $\varphi_{i}$ and $\Delta_{i}$ satisfy

$$
\begin{aligned}
& \dot{\varphi}_{1}=\Delta_{1}-K \psi_{12} \sin \varphi_{1}, \\
& \dot{\varphi}_{2}=\Delta_{2}+K\left(\psi_{12}-\psi_{13} \cos \varphi_{2}\right) \sin \varphi_{1}-K\left(\psi_{13} \cos \varphi_{1}+\psi_{23}\right) \sin \varphi_{2},
\end{aligned}
$$

and

$$
\left(\varphi_{1}, \varphi_{2}\right)(0)=\left(\varphi_{10}, \varphi_{20}\right),
$$

where $\varphi_{10}:=\theta_{10}-\theta_{20}$ and $\varphi_{20}:=\theta_{20}-\theta_{30}$.

Note that if the phase $\theta_{i}$ is strictly confined in an arc with a length $\frac{\pi}{4}$, the difference $\varphi_{i}$ is confined in a small region $\left(\left|\varphi_{i}\right| \leq c<\pi / 2\right)$. Thus we can use the above dynamics (4.3) to derive the frequency synchronization. We first show the existence of a positively invariant set for (4.3). In order to do this, in this subsection we choose $\varepsilon$ to satisfy the following conditions:

$$
\varepsilon \in\left(0, \frac{\pi}{4}\right), \quad \psi_{13} \sqrt{1-\varepsilon^{2}}+\psi_{23}>2 \sqrt{2}\left(\psi_{12}+\psi_{13}\right) \sin \varepsilon .
$$

Note that the above $\varepsilon$ does exist as long as $\left\{\psi_{i j}\right\}$ is HL. In fact, HL means that $\psi_{12}>0$ and $\psi_{13}+\psi_{23}>0$. Therefore we have the following estimate.

Lemma 4.1. Suppose that the coupling strength $K$ and initial data $\theta_{0}$ satisfy

$$
\begin{aligned}
& K>\max \left\{\frac{\left|\Delta_{1}\right|}{\psi_{12} \sin \varepsilon}, \frac{2 \sqrt{2}\left|\Delta_{2}\right|}{\psi_{13} \sqrt{1-\varepsilon^{2}}+\psi_{23}}\right\}, \\
& \theta_{i 0} \in(0, \varepsilon), \quad i=1,2,3 .
\end{aligned}
$$

Then for any solution to (4.1)-(4.2) or (4.3)-(4.4) with initial data $\theta_{0}$, we have

$$
\left|\varphi_{1}(t)\right|+\left|\varphi_{2}(t)\right|<\frac{\pi}{4}+\varepsilon, \quad t \geq 0 .
$$


Proof. Step A: Note that $\varphi_{1}=\theta_{1}-\theta_{2}$ satisfies

$$
\dot{\varphi}_{1}=\Delta_{1}-K \psi_{12} \sin \varphi_{1}, \quad \varphi_{10} \in(-\varepsilon, \varepsilon) .
$$

Then it follows from Lemma 2.5 and Remark 2.2 that

$$
\left|\varphi_{1}(t)\right| \leq \varepsilon, \quad t \geq 0 \quad \text { and } \quad\left|\varphi_{1}(t)-\varphi_{1}^{\infty}\right| \leq C e^{-\lambda_{1} t}, \quad t \gg 1,
$$

where $\varphi_{1}^{\infty}$ and $\lambda_{1}$ are given as follows.

$$
\varphi_{1}^{\infty}:=\sin ^{-1}\left(\frac{\Delta_{1}}{K \psi_{12}}\right) \in(-\varepsilon, \varepsilon), \quad \text { and } \quad \lambda_{1}:=\sqrt{\left(K \psi_{12}\right)^{2}-\Delta_{1}^{2}} .
$$

Step B: We will use the dynamics of $\left|\varphi_{2}\right|$ derived from (4.3). We first multiply the second equation in (4.3) by $\varphi_{2}$ to find

$$
\varphi_{2} \dot{\varphi}_{2}=\Delta_{2} \varphi_{2}+K \varphi_{2}\left(\psi_{12}-\psi_{13} \cos \varphi_{2}\right) \sin \varphi_{1}-K\left(\psi_{13} \cos \varphi_{1}+\psi_{23}\right) \varphi_{2} \sin \varphi_{2} .
$$

We now define a set and its supremum:

$$
\mathcal{T}_{2}:=\left\{T \in[0, \infty]:\left|\varphi_{2}(t)\right|<\frac{\pi}{4}, \quad \forall t \in[0, T)\right\}, \quad T_{2}^{*}:=\sup \mathcal{T}_{2} .
$$

Since $\left|\varphi_{20}\right|<\varepsilon<\frac{\pi}{4}$ and $\left|\varphi_{2}\right|$ is continuous, there exists $\delta_{1}>0$ such that

$$
\left|\varphi_{2}(t)\right|<\frac{\pi}{4}, \quad \forall t \in\left[0, \delta_{1}\right) .
$$

Hence $\delta_{1} \in \mathcal{T}_{2}$, i.e., $\mathcal{T}_{2}$ is not empty. We next claim that

$$
T_{2}^{*}=\infty
$$

The proof of claim: Suppose not, i.e., $T_{2}^{*}<\infty$. Then by the continuity of $\left|\varphi_{2}\right|$, there exists $\delta_{2}>0$ such that

$$
\left|\varphi_{2}(t)\right|<\frac{\pi}{2}, \quad \forall t \in\left[0, T_{2}^{*}+\delta_{2}\right) .
$$

Then, on the time interval $\left[0, T_{2}^{*}+\delta_{2}\right)$, equation (4.8) leads to an estimate as follows:

$$
\begin{aligned}
\frac{d\left|\varphi_{2}\right|}{d t} & \leq\left|\Delta_{2}\right|+K\left(\psi_{12}+\psi_{13}\right)\left|\sin \varphi_{1}\right|-K\left(\psi_{13} \cos \varphi_{1}+\psi_{23}\right) \sin \left|\varphi_{2}\right| \\
& \leq\left|\Delta_{2}\right|+K\left(\psi_{12}+\psi_{13}\right) \sin \varepsilon-K\left(\psi_{13} \sqrt{1-\varepsilon^{2}}+\psi_{23}\right) \sin \left|\varphi_{2}\right|,
\end{aligned}
$$

where we used the identity

$$
\varphi_{2} \sin \varphi_{2}=\left|\varphi_{2}\right| \sin \left|\varphi_{2}\right| \quad \text { on } \quad\left[0, T_{2}^{*}+\delta_{2}\right) .
$$

We now consider the following ODE:

$$
\dot{y}=\left|\Delta_{2}\right|+K\left(\psi_{12}+\psi_{13}\right) \sin \varepsilon-K\left(\psi_{13} \sqrt{1-\varepsilon^{2}}+\psi_{23}\right) \sin y, \quad y_{0}=\left|\varphi_{20}\right|<\varepsilon<\frac{\pi}{4} .
$$

Thanks to (4.5) and (4.6), the equilibrium $y_{e}$ of the above ODE satisfies

$$
\sin y_{e}=\frac{\left|\Delta_{2}\right|}{K\left(\psi_{13} \sqrt{1-\varepsilon^{2}}+\psi_{23}\right)}+\frac{\sin \varepsilon\left(\psi_{12}+\psi_{13}\right)}{\left(\psi_{13} \sqrt{1-\varepsilon^{2}}+\psi_{23}\right)}<\frac{1}{2 \sqrt{2}}+\frac{1}{2 \sqrt{2}}=\frac{1}{\sqrt{2}},
$$


which yields

$$
y_{e} \in\left(0, \frac{\pi}{4}\right)
$$

On the other hand, since the one-dimensional flow $y$, originating at $y(0)<\varepsilon<\frac{\pi}{4}$, always monotonically approaches the stable equilibrium solution $y_{e}$, we have

$$
y(t) \in\left[0, \frac{\pi}{4}\right), \quad t>0 .
$$

We now use (4.9) and the comparison principle of ODEs to derive

$$
\left|\varphi_{2}(t)\right| \leq y(t), \quad t \in\left[0, T_{2}^{*}+\delta_{2}\right)
$$

This yields

$$
\left|\varphi_{2}\left(T_{2}^{*}\right)\right|<\frac{\pi}{4}
$$

Again by the continuity of $\left|\varphi_{2}\right|$, there exists $\delta_{3}>0$ such that

$$
\left|\varphi_{2}(t)\right|<\frac{\pi}{4}, \quad t \in\left[0, T_{2}^{*}+\delta_{3}\right), \quad \text { i.e., } T_{2}^{*}+\delta_{3} \in \mathcal{T}_{2} .
$$

This contradicts the fact that $T_{2}^{*}=\sup \mathcal{T}_{2}$. Therefore $T_{2}^{*}=\infty$ and

$$
\left|\varphi_{2}(t)\right|<\frac{\pi}{4}, \quad t \in[0, \infty) .
$$

Finally we combine (4.7) and (4.10) to get the desired result.

Proposition 4.2. Under the same assumptions in Lemma 4.1, the system (4.1)(4.2) has ACS. More precisely, as $t \rightarrow \infty$, we have

$$
\begin{aligned}
& \text { (i) }\left|\dot{\theta}_{1}(t)-\dot{\theta}_{2}(t)\right| \leq C e^{-K\left(\psi_{12} \cos \varepsilon\right) t}, \\
& \text { (ii) }\left|\dot{\theta}_{2}(t)-\dot{\theta}_{3}(t)\right| \leq C \max \left\{e^{-K \mu t}, e^{-K \psi_{12}(\cos \varepsilon) t}\right\},
\end{aligned}
$$

where $\mu:=\psi_{13} \cos \left(\frac{\pi}{4}+\varepsilon\right)+\frac{\psi_{23}}{\sqrt{2}}$.

Proof. We set

$$
\omega_{1}:=\dot{\theta}_{1}-\dot{\theta}_{2}, \quad \omega_{2}:=\dot{\theta}_{2}-\dot{\theta}_{3},
$$

and differentiate the system (4.1) or (4.3) to get

$$
\begin{aligned}
& \dot{\omega}_{1}=-K \psi_{12}\left(\cos \varphi_{1}\right) \omega_{1}, \\
& \dot{\omega}_{2}=\left(K \psi_{12} \cos \varphi_{1}-K \psi_{13} \cos \left(\varphi_{1}+\varphi_{2}\right)\right) \omega_{1} \\
& \quad-\left(K \psi_{13} \cos \left(\varphi_{1}+\varphi_{2}\right)+K \psi_{23} \cos \varphi_{2}\right) \omega_{2} .
\end{aligned}
$$

(i) For the decay estimate of $\omega_{1}$, we use (4.7) to see

$$
\left|\varphi_{1}(t)\right| \leq \varepsilon, \quad \text { i.e., } \quad \cos \varphi_{1} \geq \cos \varepsilon, \quad t \geq 0 .
$$

This implies

$$
\omega_{1}(t) \leq \omega_{10} e^{-K\left(\psi_{12} \cos \varepsilon\right) t}, \quad t \geq 0
$$


(ii) For the decay estimate of $\omega_{2}$, we use Lemma 4.1 to see

$$
\begin{aligned}
\left|K \psi_{12} \cos \varphi_{1}-K \psi_{13} \cos \left(\varphi_{1}+\varphi_{2}\right)\right| & \leq K\left(\psi_{12}+\psi_{13}\right), \\
K \psi_{13} \cos \left(\varphi_{1}+\varphi_{2}\right)+K \psi_{23} \cos \varphi_{2} & \geq K\left[\psi_{13} \cos \left(\frac{\pi}{4}+\varepsilon\right)+\frac{\psi_{23}}{\sqrt{2}}\right], \quad t \geq 0 .
\end{aligned}
$$

In (4.11), we combine (4.12) and (4.13) to obtain

$$
\dot{\omega}_{2} \leq K\left(\psi_{12}+\psi_{13}\right) \omega_{10} e^{-K\left(\psi_{12} \cos \varepsilon\right) t}-K \mu \omega_{2}, \quad t \geq 0 .
$$

Then the standard Gronwall's lemma implies that for $t \geq 0$,

$$
\begin{aligned}
\omega_{2}(t) & \leq \omega_{2}\left(T_{0}\right) e^{-K \mu t}+\frac{\left(\psi_{12}+\psi_{13}\right) \omega_{10}}{\psi_{12} \cos \varepsilon-\mu}\left(e^{-K \psi_{12}(\cos \varepsilon) T_{0}} e^{-K \mu\left(t-T_{0}\right)}-e^{-K \psi_{12}(\cos \varepsilon) t}\right) \\
& \leq C \max \left\{e^{-K \mu t}, e^{-K \psi_{12}(\cos \varepsilon) t}\right\} .
\end{aligned}
$$

4.2. A large system with $N \geq 4$. In this part, we study ACS for a large system with $N \geq 4$.

Recall the Kuramoto model with an HL topology:

$$
\begin{aligned}
& \dot{\theta}_{1}=\Omega_{1}, \\
& \dot{\theta}_{i}=\Omega_{i}+K \sum_{j=1}^{i-1} \psi_{j i} \sin \left(\theta_{j}-\theta_{i}\right), \quad i=2, \ldots, N,
\end{aligned}
$$

subject to initial data

$$
\theta_{i}(0)=\theta_{i 0}, \quad i=1, \ldots, N
$$

Following the settings in the previous case, we set

$$
\varphi_{i}:=\theta_{i}-\theta_{i+1}, \quad \Delta_{i}:=\Omega_{i}-\Omega_{i+1}, \quad \omega_{i}:=\dot{\theta}_{i}-\dot{\theta}_{i+1}, \quad i=1,2, \ldots, N-1 .
$$

Then the $\varphi_{i}$ 's satisfy

$$
\begin{aligned}
& \dot{\varphi}_{1}=\Delta_{1}-K \psi_{12} \sin \varphi_{1}, \\
& \dot{\varphi}_{i}=\Delta_{i}+K \sum_{j=1}^{i-1} \psi_{j i} \sin \left(\sum_{l=j}^{i-1} \varphi_{l}\right)-K \sum_{j=1}^{i} \psi_{j(i+1)} \sin \left(\sum_{l=j}^{i} \varphi_{l}\right), \quad i=2, \ldots, N-1 .
\end{aligned}
$$

We define $\Gamma^{*}$ and $D_{n}$ by

$$
\begin{gathered}
\Gamma^{*}:=\max _{1 \leq l \leq N-1}\left\{\frac{\sum_{j=1}^{l-1} \psi_{j l}+\sum_{j=1}^{l} \psi_{j(l+1)}}{\sqrt{1-\left(\sin D_{\infty}\right)^{2}} \sum_{j=1}^{l-1} \psi_{j(l+1)}+\psi_{l(l+1)}}\right\}, \quad D_{\infty}<\frac{\pi}{2}, \\
S_{n}(t):=\sum_{i=1}^{n}\left|\varphi_{i}(t)\right|, \quad t \geq 0, \quad D_{n}:=\sup _{t \geq 0} S_{n}(t), \quad n=1, \ldots, N-1 .
\end{gathered}
$$


Then $D_{i}$ is an increasing sequence:

$$
D_{1} \leq D_{2} \leq \cdots \leq D_{N-1} .
$$

The following lemma presents a sufficient condition for the phases $\left\{\theta_{i}\right\}$ to remain in some region.

Lemma 4.3. Consider the Kuramoto system (4.14) with $N \geq 4$. Let $D_{\infty} \in\left(0, \frac{\pi}{2}\right)$, and let $\Gamma^{*}$ be given by (4.15). Let $\kappa \in\left(0, D_{\infty}\right)$ and $\varepsilon$ be positive constants such that

$$
\begin{aligned}
& \text { (i) }\left[\frac{\left(\Gamma^{*}+1\right)^{N-2}-1}{\Gamma^{*}}\right] \sin \kappa<\frac{1}{2} \sin D_{\infty} . \\
& \text { (ii) }\left(\Gamma^{*}+1\right)^{N-2} \sin \varepsilon<\frac{1}{2} \sin D_{\infty}, \quad \varepsilon<\frac{D_{\infty}}{N-1}, \quad \varepsilon<\kappa, \\
& \psi_{13} \sqrt{1-\varepsilon^{2}}+\psi_{23}-2 \sqrt{2}\left(\psi_{12}+\psi_{13}\right) \sin \varepsilon>0 .
\end{aligned}
$$

Suppose $\theta_{i 0} \in(0, \varepsilon), i=1, \ldots, N$, and that the coupling strength $K$ satisfies

$$
K>\max \left\{\frac{\left|\Delta_{1}\right|}{\psi_{12} \sin \varepsilon}, \max _{2 \leq l \leq N-1}\left\{\frac{\left|\Delta_{l}\right|}{\sin \kappa\left(\sqrt{1-\sin ^{2} D^{\infty}} \sum_{j=1}^{l-1} \psi_{j(l+1)}+\psi_{l(l+1)}\right)}\right\}\right\} .
$$

Then for any solution to (4.14) with initial data $\theta_{0}=\left(\theta_{10}, \ldots, \theta_{N 0}\right)$, we have

$$
\sum_{i=1}^{N-1}\left|\varphi_{i}(t)\right| \leq D_{\infty}, \quad t>0
$$

Proof. We derive a relaxation estimate for $\varphi_{i}$ inductively, i.e., we first estimate $\varphi_{1}$, and then $\varphi_{2}$, and so on.

Step A: (Estimate on $\varphi_{1}$ ). As in Step A in the proof of Lemma 4.1, we know that

$$
\left|\varphi_{1}(t)\right| \leq \varepsilon, \quad t \geq 0, \quad \text { and } \quad\left|\varphi_{1}(t)-\varphi_{1}^{\infty}\right| \leq C e^{-\lambda_{1} t}, \quad t \gg 1,
$$

where $\varphi_{1}^{\infty}$ and $\lambda_{1}$ are given as follows:

$$
\varphi_{1}^{\infty}:=\sin ^{-1}\left(\frac{\Delta_{1}}{K \psi_{12}}\right) \in(-\varepsilon, \varepsilon), \quad \text { and } \quad \lambda_{1}:=\sqrt{\left(K \psi_{12}\right)^{2}-\Delta_{1}^{2}} .
$$

Step B: (Estimate on $\varphi_{i}, i=2, \ldots, N-1$ ). Suppose that for the sub-group $\{1,2, \ldots, l\}, D_{l-1}:=\sup _{t>0} S_{l-1}(t)$ exists and

$$
D_{l-1}<D_{\infty} .
$$

We now consider the equation for $\varphi_{l}$ :

$$
\begin{aligned}
\dot{\varphi}_{l}=\Delta_{l}+ & K \sum_{j=1}^{l-1} \psi_{j l} \sin \left(\sum_{k=j}^{l-1} \varphi_{k}\right)-K \sum_{j=1}^{l} \psi_{j(l+1)} \sin \left(\sum_{k=j}^{l} \varphi_{k}\right) \\
=\Delta_{l}+ & K\left[\sum_{j=1}^{l-1} \psi_{j l} \sin \left(\sum_{k=j}^{l-1} \varphi_{k}\right)-\sum_{j=1}^{l-1} \psi_{j(l+1)} \sin \left(\sum_{k=j}^{l-1} \varphi_{k}\right) \cos \varphi_{l}\right] \\
& -K\left[\sum_{j=1}^{l-1} \psi_{j(l+1)} \cos \left(\sum_{k=j}^{l-1} \varphi_{l}\right)+\psi_{l(l+1)}\right] \sin \varphi_{l} .
\end{aligned}
$$


We multiply the above equation by $\varphi_{l}$ to get

$$
\begin{aligned}
\varphi_{l} \dot{\varphi}_{l}=\varphi_{l} \Delta_{l} & +K \varphi_{l}\left[\sum_{j=1}^{l-1} \psi_{j l} \sin \left(\sum_{k=j}^{l-1} \varphi_{k}\right)-\sum_{j=1}^{l-1} \psi_{j(l+1)} \sin \left(\sum_{k=j}^{l-1} \varphi_{k}\right) \cos \varphi_{l}\right] \\
- & K\left[\sum_{j=1}^{l-1} \psi_{j(l+1)} \cos \left(\sum_{k=j}^{l-1} \varphi_{l}\right)+\psi_{l(l+1)}\right] \varphi_{l} \sin \varphi_{l} .
\end{aligned}
$$

We define a set and its supremum:

$$
\mathcal{T}_{3}:=\left\{T \in[0, \infty]: S_{l}(t):=\sum_{i=1}^{l}\left|\varphi_{i}(t)\right|<D_{\infty}, \quad \forall t \in[0, T)\right\} \quad \text { and } \quad T_{3}^{*}:=\sup \mathcal{T}_{3} .
$$

First note that

$$
S_{l}(0)=\sum_{i=1}^{l}\left|\varphi_{i}(0)\right| \leq l \varepsilon \leq(N-1) \varepsilon<D_{\infty} .
$$

This means that there exists a $T_{0}>0$ such that

$$
\sum_{i=1}^{l}\left|\varphi_{i}(t)\right|<D_{\infty}, \quad t \in\left[0, T_{0}\right) .
$$

Thus $T_{0} \in \mathcal{T}_{3}$, i.e., $\mathcal{T}_{3}$ is not an empty set. We next claim that

$$
T_{3}^{*}=\infty \text {. }
$$

The proof of claim: Suppose not, i.e., $T_{3}^{*}<\infty$. By continuity there exists $\delta_{4}>0$ such that

$$
S_{l}(t)<\frac{\pi}{2}, \quad t \in\left[0, T_{3}^{*}+\delta_{4}\right) .
$$

Then it follows from (4.19) that

$$
\begin{aligned}
\frac{d\left|\varphi_{l}\right|}{d t} \leq\left|\Delta_{l}\right| & +K \sin S_{l-1}\left[\sum_{j=1}^{l-1} \psi_{j l}+\sum_{j=1}^{l-1} \psi_{j(l+1)}\right] \\
& -K\left[\sum_{j=1}^{l-1} \psi_{j(l+1)} \sqrt{1-\left(\sin S_{l-1}\right)^{2}}+\psi_{l(l+1)}\right] \sin \left|\varphi_{l}\right| \\
\leq\left|\Delta_{l}\right|+ & K \sin D_{l-1}\left[\sum_{j=1}^{l-1} \psi_{j l}+\sum_{j=1}^{l} \psi_{j(l+1)}\right] \\
- & K\left[\sum_{j=1}^{l-1} \psi_{j(l+1)} \sqrt{1-\left(\sin D_{\infty}\right)^{2}}+\psi_{l(l+1)}\right] \sin \left|\varphi_{l}\right|, \quad t \in\left[0, T_{3}^{*}+\delta_{4}\right)
\end{aligned}
$$

where we used

$$
\varphi_{l} \sin \varphi_{l}=\left|\varphi_{l}\right| \sin \left|\varphi_{l}\right|, \quad t \in\left[0, T_{3}^{*}+\delta_{4}\right) .
$$


Consider the following ODE:

$$
\begin{aligned}
& \dot{y}=\left|\Delta_{l}\right|+K \sin D_{l-1}\left[\sum_{j=1}^{l-1} \psi_{j l}+\sum_{j=1}^{l} \psi_{j(l+1)}\right] \\
&-K\left[\sum_{j=1}^{l-1} \psi_{j(l+1)} \sqrt{1-\left(\sin D_{\infty}\right)^{2}}+\psi_{l(l+1)}\right] \sin y, \\
& y(0)=\left|\varphi_{l 0}\right|<\varepsilon .
\end{aligned}
$$

Then by the comparison principle of ODEs, it is easy to see that

$$
\left|\varphi_{l}(t)\right| \leq y(t), \quad t \in\left[0, T_{3}^{*}+\delta_{4}\right),
$$

where $y(t)$ solves (4.20). Note that the Adler type equation (4.20) has a unique stable equilibrium $y^{\infty} \in\left(0, \frac{\pi}{2}\right)$ which is given by

$$
\sin y^{\infty}=\frac{\left|\Delta_{l}\right|}{K\left[\sqrt{1-\sin ^{2} D_{\infty}} \sum_{j=1}^{l-1} \psi_{j(l+1)}+\psi_{l(l+1)}\right]}+\frac{\sin D_{l-1}\left(\sum_{j=1}^{l-1} \psi_{j l}+\sum_{j=1}^{l} \psi_{j(l+1)}\right)}{\sqrt{1-\sin ^{2} D_{\infty} \sum_{j=1}^{l-1} \psi_{j(l+1)}+\psi_{l(l+1)}}} .
$$

Moreover, Lemma 2.5 and Remark 2.6 tell us that, $y(t)$, originating at $y(0)<\varepsilon$, monotonically approaches $y^{\infty}$ as $t \rightarrow \infty$, and therefore

$$
y(t)<\max \left\{\varepsilon, y^{\infty}\right\}, \quad t \in\left[0, T_{3}^{*}+\delta_{4}\right) .
$$

With the assumptions on $K$ and (4.15), we apply Lemma 2.2 to see that

$$
\begin{aligned}
\sin y^{\infty} & =\frac{\left|\Delta_{l}\right|}{K\left[\sqrt{1-\sin ^{2} D_{\infty}} \sum_{j=1}^{l-1} \psi_{j(l+1)}+\psi_{l(l+1)}\right]}+\frac{\sin D_{l-1}\left(\sum_{j=1}^{l-1} \psi_{j l}+\sum_{j=1}^{l} \psi_{j(l+1)}\right)}{\sqrt{1-\sin ^{2} D_{\infty}} \sum_{j=1}^{l-1} \psi_{j(l+1)}+\psi_{l(l+1)}} \\
& <\sin \kappa+\Gamma^{*} \sin D_{l-1} .
\end{aligned}
$$

We use the above relation and (4.21)-(4.22) to find

$$
\sin \left|\varphi_{l}(t)\right|<\max \left\{\sin \varepsilon, \sin \kappa+\Gamma^{*} \sin D_{l-1}\right\}=\sin \kappa+\Gamma^{*} \sin D_{l-1}, \quad t \in\left[0, T_{3}^{*}+\delta_{4}\right) .
$$

Here we used the assumption (ii) which states $\varepsilon<\kappa$. Consequently we have

$$
\begin{aligned}
\sin S_{l} & =\sin \left(\left|\varphi_{l}\right|+S_{l-1}\right)=\sin \left|\varphi_{l}\right| \cos S_{l-1}+\cos \left|\varphi_{l}\right| \sin S_{l-1} \\
& \leq \sin \left|\varphi_{l}\right|+\sin S_{l-1} \\
& <\sin \kappa+\left(\Gamma^{*}+1\right) \sin D_{l-1}, \quad t \in\left[0, T_{3}^{*}+\delta_{4}\right) .
\end{aligned}
$$

This yields

$$
\sin \left(\max _{t \in\left[0, T_{3}^{*}\right]} S_{l}(t)\right)<\sin \kappa+\left(\Gamma^{*}+1\right) \sin D_{l-1} .
$$

On the other hand, it follows from (4.16) and (4.18) that

$$
S_{i}(t)<D_{\infty}, \quad t \geq 0, \quad i=1, \ldots, l-1 .
$$


Hence the same procedure to derive (4.23) can be done inductively for $S_{i}, i=1, \ldots, l-1$ for all $t \geq 0$. Thus we have

$$
\sin S_{i}(t)<\sin \kappa+\left(\Gamma^{*}+1\right) \sin D_{i-1}, \quad i=1, \ldots, l-1, \quad t \geq 0 .
$$

This again yields

$$
\sin D_{i} \leq \sin \kappa+\left(\Gamma^{*}+1\right) \sin D_{i-1}, \quad i=1, \ldots, l-1 .
$$

We iterate the relations (4.24) and (4.25) with $\sin D_{1} \leq \sin \varepsilon$ to obtain

$$
\begin{aligned}
\sin \left(\max _{t \in\left[0, T_{3}^{*}\right]} S_{l}(t)\right) & <\left[1+\left(\Gamma^{*}+1\right)+\cdots+\left(\Gamma^{*}+1\right)^{l-2}\right] \sin \kappa+\left(\Gamma^{*}+1\right)^{l-1} \sin \varepsilon \\
& =\left[\frac{\left(\Gamma^{*}+1\right)^{l-1}-1}{\Gamma^{*}}\right] \sin \kappa+\left(\Gamma^{*}+1\right)^{l-1} \sin \varepsilon \\
& \leq\left[\frac{\left(\Gamma^{*}+1\right)^{N-2}-1}{\Gamma^{*}}\right] \sin \kappa+\left(\Gamma^{*}+1\right)^{N-2} \sin \varepsilon \\
& <\sin D_{\infty},
\end{aligned}
$$

where we used the assumptions on $\kappa$ and $\varepsilon$. Therefore we have

$$
\max _{t \in\left[0, T_{3}^{*}\right]} S_{l}(t)<D_{\infty} .
$$

By the continuity of $S_{l}$, there exists a $\delta_{5}>0$ such that

$$
S_{l}(t)<D_{\infty}, \quad t \in\left[0, T_{3}^{*}+\delta_{5}\right), \quad \text { i.e., } \quad T_{3}^{*}+\delta_{5} \in \mathcal{T}_{3} .
$$

This contradicts the fact that $T_{3}^{*}$ is the supremum of $\mathcal{T}_{3}$. Hence $T_{3}^{*}=\infty$ and we have

$$
\sum_{i=1}^{l}\left|\varphi_{i}(t)\right| \leq D_{\infty}, \quad t \geq 0 .
$$

This concludes the desired result.

REMark 4.4. The HL structure implies that $\Gamma^{*}$ is well-defined, and the right-hand side of (4.17) is finite. This means that the conditions of Lemma 4.3 are feasible.

We now give the main result in this section.

THEOREM 4.5. Under the same assumptions as in Lemma 4.2, there exist a finite number of positive constants $\left\{\mu_{i}\right\}_{i=1}^{N-1}$ satisfying

$$
\left|\dot{\theta}_{i}(t)-\dot{\theta}_{i+1}(t)\right| \leq C e^{-\mu_{i} t}, \quad t>0,
$$

i.e., exponential complete synchronization will occur asymptotically.

Proof. We use the induction method to derive the asymptotic decay of $\omega_{i}$. It follows from Lemma 4.2 that

$$
\sum_{i=1}^{N-1}\left|\varphi_{i}(t)\right| \leq D_{\infty}, \quad t>0 .
$$

- Step A (Initial step): Note that $\omega_{1}$ satisfies

$$
\frac{d}{d t} \omega_{1}=-K \psi_{12}\left(\cos \varphi_{1}\right) \omega_{1} \leq-K \psi_{12}(\cos \varepsilon) \omega_{1}
$$


Hence we have

$$
\omega_{1}(t) \leq \omega_{10} e^{-K \psi_{12}(\cos \varepsilon) t} .
$$

- Step B (Induction step): Suppose that $\omega_{i}, i \leq k-1$ satisfies

$$
\left|\omega_{i}(t)\right| \leq C e^{-\mu_{i} t}
$$

We next show that $\omega_{k}$ decays asymptotically. We differentiate the system (4.14) to derive the equation for $\omega_{k}\left(=\dot{\varphi}_{k}\right)$ :

$$
\begin{gathered}
\dot{\omega}_{k}=K \sum_{j=1}^{k-1}\left[\psi_{j k} \cos \left(\sum_{l=j}^{k-1} \varphi_{l}\right) \omega_{l}\right]-K \sum_{j=1}^{k} \psi_{j(k+1)} \cos \left(\sum_{l=j}^{k} \varphi_{l}\right) \sum_{l=j}^{k-1} \omega_{l} \\
-K \sum_{j=1}^{k} \psi_{j(k+1)} \cos \left(\sum_{l=j}^{k} \varphi_{l}\right) \omega_{k} \\
:=L\left(\omega_{1}, \omega_{2}, \ldots, \omega_{k-1}\right)-K\left[\sum_{j=1}^{k} \psi_{j(k+1)} \cos \left(\sum_{l=j}^{k} \varphi_{l}\right)\right] \omega_{k},
\end{gathered}
$$

where $L\left(\omega_{1}, \omega_{2}, \ldots, \omega_{k-1}\right)$ denotes a linear combination of $\omega_{1}, \omega_{2}, \ldots, \omega_{k-1}$ with uniformly bounded coefficients. Based on the induction hypothesis $(4.26), L\left(\omega_{1}, \omega_{2}, \ldots, \omega_{k-1}\right)$ exponentially converges to 0 :

$$
\left|L\left(\omega_{1}, \omega_{2}, \ldots, \omega_{k-1}\right)\right| \leq \mathcal{O}(1) e^{-\mu_{k-1} t}, \quad \mu_{k-1}:=\min \left\{\mu_{1}, \mu_{2}, \ldots, \mu_{k-1}\right\} .
$$

However, because $\sum_{l=j}^{k}\left|\varphi_{l}\right| \leq D_{\infty}$, the relation (4.27) yields

$$
\dot{\omega}_{k} \leq \mathcal{O}(1) e^{-\mu_{k-1} t}-K\left(\cos D_{\infty} \sum_{j=1}^{k} \psi_{j(k+1)}\right) \omega_{k} .
$$

Then we use a variant of Gronwall's lemma (see Appendix A) to find

$$
\omega_{k}(t) \leq C e^{-\mu_{k} t}, \quad \text { where } \mu_{k}:=\min \left\{\frac{\mu_{k-1}}{2}, K \cos D_{\infty} \sum_{j=1}^{k} \psi_{j(k+1)}\right\} .
$$

REMARK 4.6. If the coupling strength $K$ is sufficiently small, the ACS cannot be formed asymptotically. This can be seen easily in the three-oscillator system shown in Section 4.1. If $K<\frac{\left|\Omega_{1}-\Omega_{2}\right|}{\psi_{12}}$, then the right hand side of (4.3) cannot be zero. In this situation, $\phi:=\theta_{1}-\theta_{2}$ is either strictly increasing $\left(\Omega:=\Omega_{1}-\Omega_{2}>0\right)$ or strictly decreasing $\left(\Omega:=\Omega_{1}-\Omega_{2}<0\right)$. This means that the first two oscillators periodically meet each other and drift on the circle.

\section{Simulations}

In this section, we present several numerical simulations for the Kuramoto system with an HL topology. In all the simulations, we employed the 4-th order Runge-Kutta scheme where $N=100$. 
5.1. Identical oscillators. In this part, we consider the numerical simulations of complete synchronization for identical oscillators under HL. Without loss of generality, we choose the natural frequency to be zero. The HL topology and coupling strength $K$ are taken as follows:

$$
\psi_{j 1}=0, \quad j \geq 2, \quad \psi_{j i}=\left\{\begin{array}{cc}
\frac{1}{100}, & 1 \leq j \leq i-1, \\
0, & i+1 \leq j \leq 100 .
\end{array} \quad K=20\right.
$$

This topology means that the leader set of the $i$-th oscillator is $\mathcal{L}(i)=\{1,2, \ldots, i-1\}$. In this setting, the Kuramoto system under consideration is given by

$$
\begin{aligned}
\frac{d \theta_{1}}{d t} & =0 \\
\frac{d \theta_{i}}{d t} & =0.2 \sum_{j=1}^{i-1} \sin \left(\theta_{j}-\theta_{i}\right), \quad i=2, \ldots, 100 .
\end{aligned}
$$

The initial phases $\left\{\theta_{i 0}\right\}_{i=1}^{100}$ are generated randomly in the interval $\left(0, \frac{\pi}{2}\right)$ (see figure $5.1(\mathrm{a}))$ such that

$$
D\left(\theta_{0}\right)=1.5370<\frac{\pi}{2} .
$$

In order to make a comparison between HL and all-to-all coupling, we also use the the same initial configurations and parameters to simulate the following system:

$$
\frac{d \theta_{i}}{d t}=0.2 \sum_{j=1}^{N} \sin \left(\theta_{j}-\theta_{i}\right), \quad i=1, \ldots, 100 .
$$

\begin{tabular}{c|c|c|c|c|c}
\hline time & 0.1 & 0.2 & 0.3 & 0.4 & 0.5 \\
\hline$D(\theta)(\mathrm{HL})$ & 1.3607 & 1.2658 & 1.1791 & 1.1005 & 1.0410 \\
\hline$D(\theta)$ (all-to-all) & 0.2304 & 0.0313 & 0.0042 & $5.7514 \mathrm{e}-4$ & $7.7965 \mathrm{e}-5$ \\
\hline
\end{tabular}

TABLE 5.1. Decay of the phase diameter for identical oscillators with HL and all-to-all interactions.

The simulation results are shown in figure 5.1 and table 5.1. In particular, figure 5.1 (a) and table 5.1 show that the decay of phase diameter for the Kuramoto system under HL is much slower than that in the system with all-to-all coupling. Figure 5.1 (b) shows that the HL coupling makes the phases of all oscillators follow the fixed phase of the global leader, which explains the meaning of leadership.

\begin{tabular}{c|c|c|c|c|c|c}
\hline time & 0.1 & 0.2 & 0.3 & 0.4 & 0.5 & 0.6 \\
\hline$D(\omega)(\mathrm{HL})$ & 5.3681 & 2.7588 & 1.8659 & 1.4604 & 1.1967 & 0.9845 \\
\hline$D(\omega)$ (all-to-all) & 4.5616 & 0.6215 & 0.0843 & 0.0114 & 0.0015 & $2.1007 \mathrm{e}-4$ \\
\hline
\end{tabular}

TABLE 5.2. Decay of the frequency diameter for non-identical oscillators with HL and all-to-all interactions. 


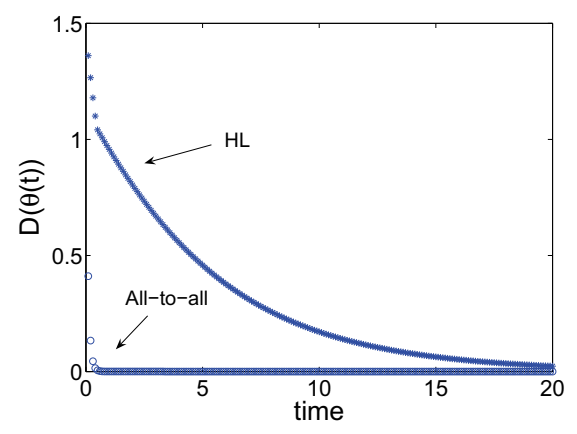

(a)

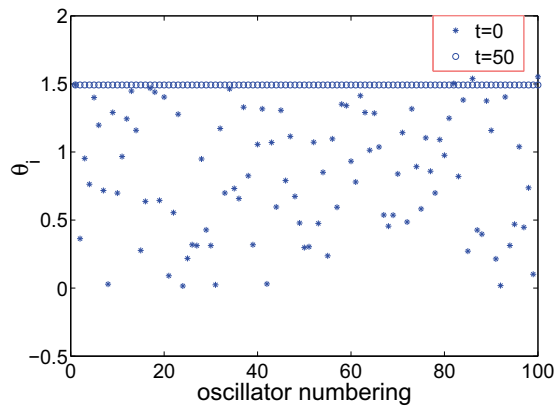

(b)

FIG. 5.1. The temporal evolution of phases for identical oscillators with the HL and all-to-all coupling.

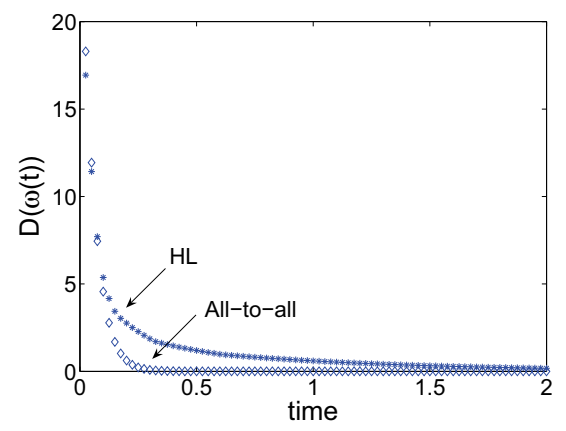

(a)

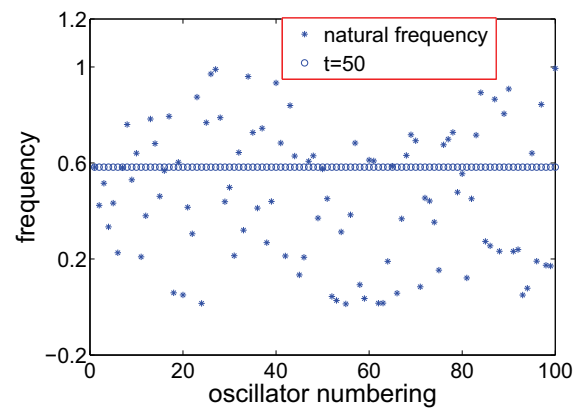

(b)

FIG. 5.2. The temporal evolution of frequency distribution for nonidentical oscillators with the $H L$ and all-to-all coupling.

5.2. Nonidentical oscillators. In this part, we present the simulation results for the Kuramoto model for nonidentical oscillators. For simplicity, we select an interaction topology (and the initial phases and parameters) to be the same as in Section 5.1, in which case the system reads

$$
\begin{aligned}
\frac{d \theta_{1}}{d t} & =\Omega_{1}, \\
\frac{d \theta_{i}}{d t} & =\Omega_{i}+0.2 \sum_{j=1}^{i-1} \sin \left(\theta_{j}-\theta_{i}\right), \quad i=2, \ldots, 100 .
\end{aligned}
$$

The natural frequencies $\Omega_{i}$ are chosen randomly and distributed uniformly in the unit interval $(0,1)$. We also use the same initial configuration and parameters to perform a simulation with an all-to-all coupling case:

$$
\frac{d \theta_{i}}{d t}=\Omega_{i}+0.2 \sum_{j=1}^{N} \sin \left(\theta_{j}-\theta_{i}\right), \quad i=1, \ldots, 100 .
$$


The simulation results are shown in figure 5.2 and table 5.2. In particular, figure 5.2 (a) and table 5.2 show that the decay of the frequency diameter for the Kuramoto system under HL structure is much slower than that of the original system with all-toall coupling structure. Figure 5.2 (b) reveals that the group of oscillators will follow upon the leader, i.e., asymptotically they have the same frequency as the leader.

\section{Conclusion}

In this study, we addressed an asymptotic complete synchronization problem for the Kuramoto oscillators with an HL topology. The HL topology is interesting because it is asymmetric, which makes it difficult to obtain any analytical results for complete synchronization. For Kuramoto oscillators over networks with symmetric topologies, the total sum of the phases is invariant up to a constant linear motion. This simple consideration is crucial if we are to perform analytical studies using an energy method. To the best of our knowledge, no systematic methodology is available for investigating the complete synchronization problem for Kuramoto oscillators in a network with an asymmetric topology. However due to the triangular structure of the adjacent coupling matrix in the interaction topology, we can use the induction method to derive exponential decay estimates for the relative frequency differences. Note that in Theorem 3.2, we see that the convergence rates toward the synchronization is nonincreasing as the size of system goes to infinity. In fact, some numerical simulations suggest "a kind of glassy behavior" in the convergence of fluctuations, which needs to be proved rigorously. We leave the rigorous verification of this phenomenon as a future project.

Appendix A. A variant of Gronwall's lemma. We next present a variant of Gronwall's lemma.

Lemma A.1. Let $y: \mathbb{R}_{+} \cup\{0\} \rightarrow \mathbb{R}_{+} \cup\{0\}$ be a differential function satisfying

$$
y^{\prime} \leq-\alpha y+f, \quad t>0, \quad y(0)=y_{0},
$$

where $\alpha$ is a positive constant and $f: \mathbb{R}_{+} \cup\{0\} \rightarrow \mathbb{R}$ is a continuous function decaying to zero, as its argument goes to infinity. Then y satisfies

$$
y(t) \leq \frac{1}{\alpha} \max _{s \in[t / 2, t]}|f(s)|+y_{0} e^{-\alpha t}+\frac{\|f\|_{L^{\infty}}}{\alpha} e^{-\frac{\alpha t}{2}}, \quad t \geq 0 .
$$

Proof. Note that $y$ satisfies

$$
y^{\prime}+\alpha y \leq f .
$$

We multiply the above differential inequality by $e^{\alpha t}$ and integrate the resulting relation from $s=0$ to $s=t$ to find

$$
\begin{aligned}
e^{\alpha t} y-y_{0} & \leq \int_{0}^{t} f(\tau) e^{\alpha \tau} d \tau \\
& =\int_{0}^{\frac{t}{2}} f(\tau) e^{\alpha \tau} d \tau+\int_{\frac{t}{2}}^{t} f(\tau) e^{\alpha \tau} d \tau \\
& \leq\|f\|_{L^{\infty}} \int_{0}^{\frac{t}{2}} e^{\alpha \tau} d \tau+\max _{\tau \in\left[\frac{t}{2}, t\right]}|f(\tau)| \int_{\frac{t}{2}}^{t} e^{\alpha \tau} d \tau \\
& \leq \frac{\|f\|_{L^{\infty}}}{\alpha}\left(e^{\frac{\alpha t}{2}}-1\right)+\frac{1}{\alpha} \max _{\tau \in\left[\frac{t}{2}, t\right]}|f(\tau)|\left(e^{\alpha t}-e^{\frac{\alpha t}{2}}\right) .
\end{aligned}
$$


Hence we have

$$
y(t) \leq \frac{1}{\alpha} \max _{\tau \in\left[\frac{t}{2}, t\right]}|f(\tau)|+\left(y_{0}-\frac{\|f\|_{L^{\infty}}}{\alpha}\right) e^{-\alpha t}+\left(\frac{\|f\|_{L^{\infty}}}{\alpha}-\frac{1}{\alpha} \max _{\tau \in\left[\frac{t}{2}, t\right]}|f(\tau)|\right) e^{-\frac{\alpha t}{2}} .
$$

Therefore for $t \geq 0$, we have

$$
y(t) \leq \frac{1}{\alpha} \max _{\tau \in\left[\frac{t}{2}, t\right]}|f(\tau)|+y_{0} e^{-\alpha t}+\frac{\|f\|_{L^{\infty}}}{\alpha} e^{-\frac{\alpha t}{2}} .
$$

Acknowledgment. Z Z. Li is indebted to Prof. Xiaoping Xue, Harbin Institute of Technology, for the suggestion of the problem. The work of S.-Y. Ha was partially supported by KRF-2011-0015388. The work of Z. Li was supported by KRF2009-0093137, NSFC grants 11126220 and 11271099, the Natural Science Foundation (A200803) of Heilongjiang Province, and the Fundamental Research Funds for the Central Universities grants HIT.KLOF.2010047 and HIT.NSRIF.2011002.

\section{REFERENCES}

[1] J.A. Acebron, L.L. Bonilla, C.J. Pérez Vicente, F. Ritort, and R. Spigler, The Kuramoto model: A simple paradigm for synchronization phenomena, Rev. Mod. Phys., 77, 137-185, 2005.

[2] D. Aeyels and J. Rogge, Stability of phase locking and existence of frequency in networks of globally coupled oscillators, Prog. Theor. Phys., 112, 921-941, 2004.

[3] R. Adler, A study of locking phenomena in oscillators, Proceedings of the I.R.E. and Waves and Electrons, 34, 351-357, 1946.

[4] A. Arenas, A. Díaz-Guilera, J. Kurths, Y. Moreno, and C. Zhou, Synchronization in complex networks, Phys. Rep., 469, 93-153, 2008.

[5] J. Buck and E. Buck, Biology of sychronous flashing of fireflies, Nature, 211, 562-564, 1966.

[6] L.L. Bonilla, J.C. Neu, and R. Spigler, Nonlinear stability of incoherence and collective synchronization in a population of coupled oscillators, J. Stat. Phy., 67, 313-330, 1992.

[7] N.J. Balmforth and R. Sassi, A shocking display of synchrony, Physica D, 143, 21-55, 2000.

[8] Y. Choi, S.Y. Ha, S. Jung, and Y. Kim, Asymptotic formation and orbital stability of phaselocked states for the Kuramoto model, Physica D, 241, 735-754, 2012.

[9] Y.P. Choi, S.Y. Ha, and S.B. Yun, Complete synchronization of Kuramoto oscillators with finite inertia, Physica D, 240, 32-44, 2011.

[10] N. Chopra and M.W. Spong, On exponential synchronization of Kuramoto oscillators, IEEE Trans. Automatic Control, 54, 353-357, 2009.

[11] F. Cucker and J.G. Dong, On the critical exponent for flocks under hierarchical leadership, M3AS., 19, 1391-1404, 2009.

[12] F. Cucker and S. Smale, Emergent behavior in flocks, IEEE Trans. Automat. Control, 52, 852-862, 2007.

[13] F. De Smet and D. Aeyels, Partial entrainment in the finite Kuramoto-Sakaguchi model, Physica D, 234, 81-89, 2007.

[14] F. Dörfler and F. Bullo, On the critical coupling for Kuramoto oscillators, SIAM. J. Appl. Dyn. Syst., 10, 1070-1099, 2011.

[15] F. Dörfler and F. Bullo, Synchronization and transient stability in power networks and nonuniform Kuramoto oscillators, SIAM. J. Contr. Optim., 50, 1616-1642, 2012.

[16] G.B. Ermentrout, Synchronization in a pool of mutually coupled oscillators with random frequencies, J. Math. Biol., 22, 1-9, 1985.

[17] S.Y. Ha, T. Ha, and J.H. Kim, On the complete synchronization of the Kuramoto phase model, Physica D, 239, 1692-1700, 2010.

[18] S.Y. Ha, E. Jeong, and M.J. Kang, Emergent behaviour of a generalized Vicsek-type flocking model, Nonlin., 23, 3139-3156, 2010.

[19] S.Y. Ha and M.J. Kang, Fast and slow relaxations to bi-cluster configurations for the ensemble of Kuramoto oscillators, Quart. Appl. Math., to appear.

[20] S.Y. Ha, C. Lattanzio, B. Rubino, and M. Slemrod, Flocking and synchronization of particle models, Quart. Appl. Math., 69, 91-103, 2011. 
[21] S.Y. Ha and M. Slemrod, A fast-slow dynamical systems theory for the Kuramoto phase model, J. Diff. Equ., 251, 2685-2695, 2011.

[22] A. Jadbabaie, N. Motee, and M. Barahona, On the stability of the Kuramoto model of coupled nonlinear oscillators, Proceedings of the American Control Conference, Boston Massachusetts, 2004.

[23] Y. Kuramoto, Chemical Oscillations, Waves and Turbulence, Springer-Verlag Berlin, 1984.

[24] Y. Kuramoto, International Symposium on Mathematical Problems in Mathematical Physics, Lecture notes in Theoretical Physics, 30, 420, 1975.

[25] Z. Li and X. Xue, Cucker-Smale flocking under rooted leadership with fixed and switching topologies, SIAM J. Appl. Math., 70, 3156-3174, 2010.

[26] Z. Lin, B. Francis, and M. Maggiore, State agreement for continuous-time coupled nonlinear systems, SIAM J. Contr. Optim., 46, 288-307, 2008.

[27] R.E. Mirollo and S.H. Strogatz, The spectrum of the partially locked state for the Kuramoto model of coupled oscillator, J. Nonlin. Sci., 17, 309-347, 2007.

[28] R.E. Mirollo and S.H. Strogatz, The spectrum of the locked state for the Kuramoto model of coupled oscillator, Physica D, 205, 249-266, 2005.

[29] R.E. Mirollo and S.H. Strogatz, Stability of incoherence in a populations of coupled oscillators, J. Stat. Phy., 63, 613-635, 1991.

[30] L. Moreau, Stability of multiagent systems with time-dependent communication links, IEEE Trans. Autom. Control, 50, 169-182, 2005.

[31] A. Pikovsky, M. Rosenblum, and J. Kurths, Synchrnization: A Universal Concept in Nonlinear Sciences, Cambridge University Press, Cambridge, 2001.

[32] J.G. Restrepo, E. Ott, and B.R. Hunt, Synchronization in large directed networks of coupled phase oscillators, Chaos, 16, 015107, 2005.

[33] A. Sarlette, Geometry and Symmetries in Coordination Control, PhD Thesis at University of Liege, 2009.

[34] J. Shen, Cucker-Smale flocking under hierarchical leadership, SIAM J. Appl. Math., 68, 694719, 2007.

[35] P.S. Skarda, E. Ott, and J.G. Restrepo, Cluster synchrony in systems of coupled phase oscillators with higher-order coupling, Phys. Rev. E, 84, 036208, 2011.

[36] S.H. Strogatz, From Kuramoto to Crawford: Exploring the onset of synchronization in populations of coupled oscillators, Physica D, 143, 1-20, 2000.

[37] J.L. van Hemmen and W.F. Wreszinski, Lyapunov function for the Kuramoto model of nonlinearly coupled oscillators, J. Stat. Phys., 72, 145-166, 1993.

[38] A.T. Winfree, The Geometry of Biological Time, Springer New York, 1980.

[39] A.T. Winfree, Biological rhythms and the behavior of populations of coupled oscillators, J. Theor. Biol., 16, 15-42, 1967. 